\title{
POSITIVE DEFINITE MEASURES
}

ROBERT J. BLATTNER ${ }^{1}$

In this paper we prove two theorems relating positive definite measures to induced representations. The first shows how the injection of a positive definite measure on a topological group $H$ into a containing locally compact group $G$ in which $H$ is closed gives rise to induced representations. The second is another version of Mackey's imprimitivity theorem, along the lines of Loomis' proof [5]. We feel this is justified on several grounds. Firstly, our proof is simpler than Loomis'. We make no use of the Radon-Nikodym theorem nor of quasi-invariant measures. Secondly, we do not assume in advance that our system of imprimitivity is based on the reduced algebra of Borel sets in $G / H$. Instead, this fact is seen as a consequence of the theorem. Finally, the statement and proof of Theorem 2 in [5] are in need of minor repairs. Using Loomis' notation, the induced representation space of $V$ is spanned, not by the set of functions $\left\{f_{u}: u \in H\right\}$, but rather by the set $\left\{[E] f_{u}: u \in H, E\right.$ a Borel subset of $\left.G / K\right\}$. Formula (8) must then be replaced by formula (11) in the statement of the theorem. The algebra $C_{0}(S \times G)$ used in the present paper may be looked upon as a device for accomplishing these changes.

All nonobvious definitions, notations, and conventions are those of $[1]$.

1. Let $f, g \in C_{0}(G)$. Define $f \circ g$ and $f^{*}$ by

$$
(f \circ g)(x)=\int f(y) g\left(x y^{-1}\right) d y
$$

and

$$
f^{*}(x)=\left[f\left(x^{-1}\right)\right]^{-} \delta_{G}(x)^{-1} .
$$

$C_{0}(G)$, equipped with $\circ,{ }^{*}$, and the usual inductive limit topology, is a topological *-algebra. This is a group algebra with multiplication defined in a way differing slightly from the usual one. If $x \in G$, we define $(R(x) f)(y)=f(y x)$. The map $(x, f) \rightarrow R(x) f$ is continuous.

A measure $\mu$ on $G$ such that $\mu\left(f^{*} \circ f\right) \geqq 0$ for all $f \in C_{0}(G)$ is called positive definite. Given such a $\mu$, one defines a pseudo-Hilbert inner product on $C_{0}(G)$ by setting $(f, g)_{\mu}=\mu\left(g^{*} \circ f\right)$. One then completes

Received by the editors March 28, 1962.

${ }^{1}$ National Science Foundation Fellow. 
$C_{0}(G)$ to get a Hilbert space $\mathcal{H}_{\mu}$ and, for each $x \in G$, extends $R(x)$ to a unitary operator $R_{\mu}(x)$ on $\mathfrak{H}_{\mu} . R_{\mu}$ is then a unitary representation of $G$ on $\mathcal{F}_{\mu}$.

THEOREM 1. Let $H$ be a closed subgroup of the locally compact group $G$. Let $\mu$ be a positive definite measure on $H$. Let $\nu$ be the measure on $G$ obtained by injecting $\delta_{H}^{-1 / 2} \delta_{G}^{1 / 2} \mu$. Then $\nu$ is positive definite. Moreover, $R_{\nu}$ is unitarily equivalent to $U^{R_{\mu}}$ via the closure $V$ of the map $f \rightarrow \tilde{f}$ of $C_{0}(G)$, where, for $x \in G, \tilde{f}(x)$ is the vector in $\mathfrak{H}_{\mu}$ defined by $\tilde{f}(x)(\xi)$ $=\delta_{H}(\xi)^{-1 / 2} \delta_{G}(\xi)^{1 / 2} f(\xi x)$.

Proof. Let $f \in C_{0}(G)$ and choose $h \geqq 0$ in $C_{0}(G)$ such that $\int_{H} h(\xi x) d \xi$ $=1$ for all $x \in G$ such that $f(x) \neq 0$. Then

$$
\begin{aligned}
\int\left(f^{*} \circ f\right)(\xi) \delta_{H}(\xi)^{-1 / 2} \delta_{G}(\xi)^{1 / 2} d \mu(\xi) \\
\quad=\iint[f(x)]^{-} f(\xi x) \delta_{H}(\xi)^{-1 / 2} \delta_{G}(\xi)^{1 / 2} d x d \mu(\xi) \\
\quad=\iiint h(\eta x)[f(x)]^{-} f(\xi x) \delta_{H}(\xi)^{-1 / 2} \delta_{G}(\xi)^{1 / 2} d \eta d x d \mu(\xi) \\
=\iiint h(x)\left[f\left(\eta^{-1} x\right)\right]^{-} f\left(\xi \eta^{-1} x\right) \delta_{H}(\xi)^{-1 / 2} \delta_{G}(\xi)^{1 / 2} \delta_{G}(\eta)^{-1} d \eta d x d \mu(\xi) \\
=\iiint h(x)[f(\eta x)]^{-} f(\xi \eta x) \delta_{H}(\xi)^{-1 / 2} \delta_{G}(\xi)^{1 / 2} \delta_{H}(\eta)^{-1} \delta_{G}(\eta) d \eta d x d \mu(\xi) \\
=\iiint h(x)[\tilde{f}(x)(\eta)]-\tilde{f}(x)(\xi \eta) d \eta d \mu(\xi) d x \\
=\int h(x)(\tilde{f}(x), \tilde{f}(x))_{\mu} d x \geqq 0 .
\end{aligned}
$$

Thus $\nu$ is positive definite.

It is trivial to verify that $\tilde{f} \in \mathcal{F}_{0}$ (see $[1, \S 2]$ for the definition). Therefore $\tilde{f}$ is in the Hilbert space $\mathfrak{F C}$ of $U^{R_{\mu}}$. Moreover, the above equations, together with the definition of the norm in $\mathfrak{H}$, show that $\|f\|_{\nu}=\|\tilde{f}\|$. Hence the isometry $V$ is well defined. Since $V$ clearly sets up an equivalence between $R_{\nu}$ and a subrepresentation of $U^{R_{\mu}}$, we only have left to show that $V$ is onto. form

Let $g \in C_{0}(G), u \in C_{0}(H)$. Regarding $u$ as a member of $\mathcal{H}_{\mu}$, we may

$$
\epsilon(g, u)(x)=\int_{H} \delta_{H}(\xi)^{-1 / 2} \delta_{G}(\xi)^{1 / 2} g(\xi x) R_{\mu}(\xi)^{-1} u d \xi
$$


as in [1]. Since this integral converges in $C_{0}(H)$, we obtain

$$
\begin{aligned}
\epsilon(g, u)(x)(\eta) & =\int_{H} \delta_{H}(\xi)^{-1 / 2} \delta_{G}(\xi)^{1 / 2} g(\xi x) u\left(\eta \xi^{-1}\right) d \xi \\
& =\int_{I I} \delta_{H}(\xi \eta)^{-1 / 2} \delta_{G}(\xi \eta)^{1 / 2} g(\xi \eta x) u\left(\xi^{-1}\right) d \xi .
\end{aligned}
$$

It is now easy to see that if we set $f(x)=\int_{H} \delta_{H}(\xi)^{-1 / 2} \delta_{G}(\xi)^{1 / 2} g(\xi x) u\left(\xi^{-1}\right) d \xi$, then $f \in C_{0}(G)$ and $\tilde{f}=\epsilon(g, u)$. Thus $\left[\tilde{f}: f \in C_{0}(G)\right]$ is dense in $\mathcal{H C}$ by $[1$, Lemma $2 b]$, and $V$ is onto.

2. Let $(S, G)$ be a locally compact transformation group (with $G$ acting on the right). By a unitary representation of $(S, G)$ on the Hilbert space $\mathfrak{H C}$ we shall mean a ${ }^{*}$-representation $E$ of $C_{0}(S)$ (under the pointwise operations) in $\mathscr{L}(\mathfrak{F}, \mathfrak{F})$ together with a unitary representation $U$ of $G$ on $\mathcal{H}$ such that:

(1) $E\left(C_{0}(S)\right) \mathcal{H}$ is dense in $\mathfrak{H}$;

(2) $U(x) E(f) U\left(x^{-1}\right)=E(R(x) f), x \in G$, where $(R(x) f)(p)=f(p x)$.

Note that from the ${ }^{*}$-representation property of $E$ it follows that $E$ is continuous from $C_{0}(S)$ in the $\|\cdot\|_{\infty}$ norm to $\mathcal{L}(\mathcal{F C}, \mathfrak{F C})$ in the uniform norm.

As an example, let $G$ be a locally compact group and $H$ a closed subgroup. Let $S=G / H$ (right cosets) and let $G$ operate on $S$ in the usual way. Let $\pi$ be the canonical projection of $G$ onto $S$. Let $L$ be a unitary representation of $H$. Form the induced representation $U^{L}$ of $G$, operating on the Hilbert space of functions $\mathcal{H}$. For $f \in C_{0}(S)$, define $E^{L}(f)$ on $\mathcal{H C}$ by setting $\left(E^{L}(f) g\right)(x)=f(\pi(x)) g(x)$. It is easily verified that this definition makes sense and that $\left(E^{L}, U^{L}\right)$ is a unitary representation of $(S, G)$ on $\mathcal{H}$. It is called the unitary representation of $(S, G)$ induced by $L$.

Returning now to a general transformation group, let $f, g$ $\in C_{0}(S \times G)$. Define $f \circ g$ and $f^{*}$ by $(f \circ g)(p, x)=\int f(p, y) g\left(p y^{-1}, x y^{-1}\right) d y$ and $f^{*}(p, x)=\left[f\left(p x^{-1}, x^{-1}\right)\right]^{-} \delta_{G}(x)^{-1}$. It is easily verified that $\circ$ and ${ }^{*}$ turn $C_{0}(S \times G)$ into a topological *-algebra (with respect to the usual inductive limit topology on $\left.C_{0}(S \times G)\right)$. Moreover, if $x \in G$ we define $(R(x) f)(p, y)=f(p x, y x)$, and if $h \in C_{0}(S)$ we define $(P(h) f)(p, x)$ $=h(p) f(p, x)$. It is easy to see that $(x, f) \rightarrow R(x) f$ and $(h, f) \rightarrow P(h) f$ are continuous maps from $G \times C_{0}(S \times G)$ into $C_{0}(S \times G)$ and $C_{0}(S)$ $\times C_{0}(S \times G)$ into $C_{0}(S \times G)$ respectively (even when $C_{0}(S)$ is given the sup topology). The algebra $C_{0}(S \times G)$ is due to Dixmier [3] and has been studied extensively by Glimm [4]. 
Let $(E, U)$ be a unitary representation of $(S, G)$ on $\mathfrak{F}$. For $f \in C_{0}(S \times G)$ define $\Phi(f)$ by $\Phi(f)=\int E(f(\cdot, x)) U\left(x^{-1}\right) d x$. It is easily verified that $\Phi$ is a continuous *-homomorphism from $C_{0}(S \times G)$ into $\mathscr{L}(\mathfrak{H}, \mathfrak{H C})$ such that $\Phi\left(C_{0}(S \times G)\right) \mathcal{H C}$ is dense in $\mathcal{H C}$ and such that $\Phi(R(x) f)=U(x) \Phi(f)$ and $\Phi(P(h) f)=E(h) \Phi(f)$ for all $x \in G$ and $h \in C_{0}(S)$. Let $v \in \mathfrak{F C}$ and define $\Lambda$ by $\Lambda(f)=(\Phi(f) v, v) . \Lambda$ is a Radon measure on $S \times G$ such that $\Lambda\left(f^{*} \circ f\right) \geqq 0$ for all $f \in C_{0}(S \times G)$. Any measure on $S \times G$ satisfying this positivity condition will be called positive definite.

Let $\Lambda$ be a positive definite measure on $S \times G$. Exactly as in the case of positive definite measures on groups, we may define a pseudoHilbert inner production on $C_{0}(S \times G)$ by setting $(f, g)_{\Lambda}=\Lambda\left(g^{*} \circ f\right)$. We may then complete $C_{0}(S \times G)$ to get a Hilbert space $\kappa_{\Lambda}$. For $x \in G, R(x)$ extends to a unitary operator $R_{\Lambda}(x)$ on $\mathfrak{K}_{\Lambda}$; for $h \in C_{0}(S)$, $P(h)$ extends to a bounded operator $P_{\Lambda}(h)$ on $\mathcal{K}_{\Lambda} .\left(P_{\Lambda}, R_{\Lambda}\right)$ is a unitary representation of $(S, G)$ on $\mathcal{K}_{\Lambda}$. If, moreover, $\Lambda$ arises from a unitary representation $(E, U)$ of $(S, G)$ on $\mathfrak{H}$ and a vector $v \in \mathcal{H C}$, as above, and if $\mathcal{H}_{1}$ is the smallest $(E, U)$-invariant subspace of $\mathfrak{H C}$ containing $v$, then $\left(P_{\Lambda}, R_{\Lambda}\right)$ is unitarily equivalent to the restriction $(E, U) \mid \mathfrak{F}_{1}$ of $(E, U)$ to $\mathfrak{F}_{1}$ via the closure of the isometry $f \rightarrow \Phi(f)$ v.

Suppose now that $H$ is a closed subgroup of $G$ and that $S=G / H$. For $h \in C_{0}(G)$, set

$$
(\tau h)(\pi(x))=\int_{H} h(\xi x) d \xi .
$$

For $k \in C_{0}(G \times G \times G)$, set $(\sigma k)(\pi(x), y, z)=\int_{H} k(\xi x, y, z) d \xi$ and $(\theta k)(x, y, z)=k\left(x z, y x^{-1}, x^{-1}\right)$. Then $\tau$ and $\sigma$ are open homomorphisms of $C_{0}(G)$ and $C_{0}(G \times G \times G)$ onto $C_{0}(S)$ and $C_{0}(S \times G \times G)$ respectively, and $\theta$ is a topological automorphism of $C_{0}(G \times G \times G)$.

Lemma. Let $\Lambda$ be a measure on $S \times G$. Define the measure $\mathbf{M}$ on $G \times G \times G$ by setting

$$
\iiint k(x, y, z) d \mathrm{M}(x, y, z)=\iiint(\sigma \theta k)(p, y, z) d \Lambda(p, y) d z
$$

for all $k \in C_{0}(G \times G \times G)$. Then there is a measure $\mu$ on $G \times G$ such that $d \mathbf{M}(x, y, z)=d x d \mu(y, z)$. Moreover, for $\xi \in H, d \mu(y \xi, z \xi)$ $=\delta_{G}(\xi) \delta_{H}(\xi)^{-1} d \mu(y, z)$.

Proof. That $M$ factors as above follows from the fact that $M$ is invariant under right translation in its first variable (cf. the argument in [2, bottom of p. 127]). Now let $k \in C_{0}(G \times G \times G)$ and $\xi \in H$. Set $k^{\xi}(x, y, z)=k\left(x, y \xi^{-1}, z \xi^{-1}\right)$ and $k_{\xi}(x, y, z)=k\left(\xi^{-1} x, y, z\right)$. Then 


$$
\begin{aligned}
\left(\sigma \theta k^{\xi}\right)(\pi(x), y, z) & =\int_{H} k\left(\eta x z, y x^{-1} \eta^{-1} \xi^{-1}, x^{-1} \eta^{-1} \xi^{-1}\right) d \eta \\
& =\delta_{H}(\xi)^{-1} \int_{H} k\left(\xi^{-1} \eta x, y x^{-1} \eta^{-1}, x^{-1} \eta^{-1}\right) d \eta \\
& =\delta_{H}(\xi)^{-1}\left(\sigma \theta k_{\xi}\right)(\pi(x), y, z) .
\end{aligned}
$$

From this we obtain

$$
\begin{aligned}
\iiint k(x, y, z) d x d \mu(y \xi, z \xi) & =\iiint k^{\xi}(x, y, z) d x d \mu(y, z) \\
& =\delta_{H}(\xi)^{-1} \iiint k_{\xi}(x, y, z) d x d \mu(y, z) \\
& =\delta_{H}(\xi)^{-1} \delta_{G}(\xi) \iiint k(x, y, z) d x d \mu(y, z)
\end{aligned}
$$

and our lemma is proved.

Theorem 2. Let $\Lambda$ be a positive definite measure on $S \times G$ and define $\mu$ as in the lemma. For $\phi, \psi \in C_{0}(G)$, set $(\phi, \psi)_{\mu}=\int \phi(y)[\psi(z)]-d \mu(y, z)$. If $\xi \in H$, set $(L(\xi) \phi)(y)=\delta_{G}(\xi)^{1 / 2} \delta_{H}(\xi)^{-1 / 2} \phi(y \xi)$. Then $(\cdot, \cdot)_{\mu}$ is a pseudo-Hilbert inner production on $C_{0}(G)$. Complete $C_{0}(G)$ to get the Hilbert space $V_{\mu}$. Then $L$ extends to a unitary representation $L_{\mu}$ of $H$ on $v_{\mu}$. Finally $\left(P_{\Lambda}, R_{\Lambda}\right)$ is unitarily equivalent to $\left(E^{L_{\mu}}, U^{L_{\mu}}\right)$ via the closure $W$ of map $f \rightarrow \hat{f}$ of $C_{0}(S \times G)$, where, for $x \in G, \hat{f}(x)$ is the vector in $\vartheta_{\mu}$ defined by $\hat{f}(x)(y)=f(\pi(x), y x)$.

Proof. Let $f \in C_{0}(S \times G)$ and $h \in C_{0}(G)$. Set $k(x, y, z)$ $=h(x)[f(\pi(x), z x)]-f(\pi(x), y x)$. Then

$$
\begin{aligned}
(\sigma \theta k)(\pi(x), y, z) & =\int_{H} h(\xi x z)[f(\pi(x z), z)]-f(\pi(x z), y z) d \xi \\
& =(\tau h)(\pi(x z))[f(\pi(x z), z)]-f(\pi(x z), y z) .
\end{aligned}
$$

Hence

$$
\begin{aligned}
\int h(x)(\hat{f}(x), \hat{f}(x))_{\mu} d x & =\iiint(\tau h)(p z)[f(p z, z)]-f(p z, y z) d z d \Lambda(p, y) \\
& =\iiint f^{*}(p, z)(\tau h)\left(p z^{-1}\right) f\left(p z^{-1}, y z^{-1}\right) d z d \Lambda(p, y) \\
& =\Lambda\left(f^{*} \circ P(\tau h) f\right)=\left(P_{\Lambda}(\tau h) f, f\right)_{\Delta} .
\end{aligned}
$$


Now $h \geqq 0$ implies that $\tau h \geqq 0$, so that $P_{\Delta}(\tau h)$ is a positive operator. Moreover, $x \rightarrow \hat{f}(x)[\hat{f}(x)]-$ is continuous from $x$ to $C_{0}(G)$ so that $x \rightarrow(\hat{f}(x), \hat{f}(x))_{\mu}$ is a continuous function. We conclude that $(\hat{f}(x), \hat{f}(x))_{\mu}$ $\geqq 0$ for all $x \in G$. Since $f \rightarrow \hat{f}(e)$ maps $C_{0}(S \times G)$ onto $C_{0}(G)$, our first assertion is proved. That $L(\xi)$ is unitary for all $\xi \in H$ follows from the lemma, and that $L_{\mu}$ is a unitary representation of $H$ is then clear.

Now it is easy to see that $\hat{f} \in F_{0}$. Choose $h \in C_{0}(G)$ so that $\tau h=1$ on $[p \in S: f(p, y) \neq 0$ for some $y \in G]$. Then $P_{\Delta}(\tau h) f=f$ and we obtain $\|f\|_{\Lambda}=\|\hat{f}\|$. Once again we are reduced to showing that $W$ is onto. This is done exactly as in Theorem 1. Let $g, u \in C_{0}(G)$. Regarding $u$ as a member of $\mho_{\mu}$, form $\epsilon(g, u)$. We obtain $\epsilon(g, u)(x)(y)$ $=\int_{H} g(\xi x) u\left(y \xi^{-1}\right) d \xi$. Set $f(\pi(x), y)=\int_{H} g(\xi x) u\left(y x^{-1} \xi^{-1}\right) d \xi$. If the supports of $g$ and $u$ are $K_{1}$ and $K_{2}$ respectively, the support of $f$ is contained in $\pi\left(K_{1}\right) \times\left(K_{2} K_{1}\right)$, compact. Hence $f \in C_{0}(S \times G)$. It is easy to see that $\hat{f}=\epsilon(g, u)$, and our proof finishes as before.

CoROLlaRy. Every unitary representation of $(G / H, G)$ is induced.

Proof. If the representation space is jointly cyclic under $E$ and $U^{\prime}$ the corollary follows from the theorem together with the remarks two paragraphs before the lemma. The general case follows from the fact that induction commutes with direct summation.

\section{REFERENCES}

1. R. J. Blattner, On induced representations, Amer. J. Math. 83 (1961), 79-98.

2. F. Bruhat, Sur les représentations induites des groupes de Lie, Bull. Soc. Math. France 84 (1956), 97-205.

3. J. Dixmier, Algèbres quasi-unitaires, Comment. Math. Helv. 26 (1952), 275321.

4. J. Glimm, Families of induced representations, Pacific J. Math. 12 (1962), 885911.

5. L. H. Loomis, Positive definite functions and induced representations, Duke Math. J. 27 (1960), 569-579.

University of California, Los Angeles 